Research Article

\title{
Blow-Up Results for a Class of Quasilinear Parabolic Equation with Power Nonlinearity and Nonlocal Source
}

\author{
Xiaorong Zhang $\mathbb{D}$ and Zhoujin Cui \\ School of Economics and Management, Jiangsu Maritime Institute, Nanjing 211170, China \\ Correspondence should be addressed to Zhoujin Cui; cuizhoujin@126.com
}

Received 14 August 2021; Accepted 8 October 2021; Published 22 October 2021

Academic Editor: Feliz Minhos

Copyright (c) 2021 Xiaorong Zhang and Zhoujin Cui. This is an open access article distributed under the Creative Commons Attribution License, which permits unrestricted use, distribution, and reproduction in any medium, provided the original work is properly cited.

This paper deals with a class of quasilinear parabolic equation with power nonlinearity and nonlocal source under homogeneous Dirichlet boundary condition in a smooth bounded domain; we obtain the blow-up condition and blow-up results under the condition of nonpositive initial energy.

\section{Introduction}

In this paper, we consider the following quasilinear parabolic equation with power nonlinearity and nonlocal source term:

$$
\begin{cases}u_{t}=\Delta_{p} u+\mu u^{p} \int_{\Omega} u^{p+1}(y, t) d y-k|u|^{p-2} u, & (x, t) \in \Omega \times(0, T), \\ u(x, t)=0, & (x, t) \in \partial \Omega \times(0, T), \\ u(x, 0)=u_{0}(x), & x \in \Omega,\end{cases}
$$

where $\Omega \subset R^{N}(N \geq 1)$ is a bounded domain with smooth boundary $\partial \Omega$ and $\Delta_{p} u=\operatorname{div}\left(|\nabla u|^{p-2} \nabla u\right)$ is the standard $p$-Laplace operator with $p>2, \mu, k>0, u_{0}(x) \in W_{0}^{1, p}(\Omega) \backslash\{0\}$.

In the past decades, many physical phenomena have been expressed as nonlocal mathematical models (see [1, $2]$ ). It is also suggested that the nonlocal growth term provides a more realistic model for the physical model of compressible reaction gas. Problem (1) appears in the study of fluid flow through porous media with integral source (see $[3,4]$ ) and population dynamics (see $[5,6]$ ). Actually, equations of the above form are mathematical models occurring in studies of the $p$-Laplace equation ([7-15] and references therein), generalized reaction-diffusion theory [16], nonNewtonian fluid theory $[17,18]$, non-Newtonian filtration theory $[19,20]$, and the turbulent flow of a gas in porous medium [7]. Media with $p>2$ are called dilatant fluids and those with $p<2$ are called pseudoplastics. If $p=2$, they are Newtonian fluids. When $p \neq 2$, the problem becomes more complicated since certain nice properties inherent to the case $p=2$ seem to be lose or at least difficult to verify.

Blow-up results of parabolic equations with nonlocal sources have been studied as well. For example, the problem of the form

$$
\begin{cases}u_{t}=\operatorname{div}\left(|\nabla u|^{p-2} \nabla u\right)+\int_{\Omega} u^{q} d x, & (x, t) \in \Omega \times(0, T) \\ u(x, t)=0, & (x, t) \in \partial \Omega \times(0, T) \\ u(x, 0)=u_{0}(x), & x \in \Omega,\end{cases}
$$

was studied by Li and Xie [7]. They established global existence of solutions and discussed the blow-up properties of solutions. 
The authors in [8] studied the following $p$-Laplacian equation with power nonlinearity

$$
\begin{cases}u_{t}-\operatorname{div}\left(|\nabla u|^{p-2} \nabla u\right)+|u|^{p-2} u=|u|^{p-2} u \ln |u|, & x \in \Omega, t>0, \\ u(x, t)=0, & (x, t) \in \partial \Omega \times(0, T), \\ u(x, 0)=u_{0}(x), & x \in \Omega .\end{cases}
$$

By using an efficient technique and according to some sufficient conditions, the global existence and decay estimates of solutions under some sufficient conditions are discussed.

The authors in [9] considered the Neumann problem to the following initial parabolic equation with logarithmic source:

$$
\begin{cases}u_{t}-\operatorname{div}\left(|\nabla u|^{p-2} \nabla u\right)=|u|^{p-2} u \log |u|-\oint_{\Omega}|u|^{p-2} u \log |u| d x, & x \in \Omega, t>0, \\ \frac{\partial u(x, t)}{\partial \eta}=0, & x \in \partial \Omega, t>0, \\ u(x, 0)=u_{0}(x), & x \in \Omega,\end{cases}
$$

in a bounded domain with smooth boundary, $p>2$. By using the logarithmic Sobolev inequality and potential wells method, they obtain the decay, blow-up, and nonextinction of solutions under some conditions.

In [10], the following model of a quasilinear diffusion equation with interior logarithmic source has been studied:

$$
\begin{cases}u_{t}-\operatorname{div}\left(|\nabla u|^{p-2} \nabla u\right)=|u|^{p-2} u \log |u|, & x \in \Omega, t>0, \\ u(x, t)=0, & x \in \partial \Omega, t>0, \\ u(x, 0)=u_{0}(x), & x \in \Omega,\end{cases}
$$

in which $p>2, u_{0}(x) \in W_{0}^{1, p}(\Omega) \backslash\{0\}$. By using the potential well method and a logarithmic Sobolev inequality, the authors obtained results of existence or nonexistence of global weak solution. They also provided sufficient conditions for the large time decay of global weak solutions and for the finite time blow-up of weak solutions. Among some other interesting results, they showed that the weak solution $u(x, t)$ of problem (5) blows up at finite time under the condition $J\left(u_{0}\right) \leq M$ and $I\left(u_{0}\right)<0$, where $M>0$ is a constant; the energy functional $J(u)$ and Nehari functional $I(u)$ are defined as follows:

$$
\begin{aligned}
& J(u)=\frac{1}{p}\|\nabla u\|_{p}^{p}-\frac{1}{p} \int_{\Omega}|u|^{p} \log |u| d x+\frac{1}{p^{2}}\|u\|_{p}^{p}, \\
& I(u)=\|\nabla u\|_{p}^{p}-\int_{\Omega}|u|^{p} \log |u| d x,
\end{aligned}
$$

in which $\|\cdot\|_{p}=\left(\int_{\Omega}|\cdot|^{p} d x\right)^{1 / p}$.

Motivated by the above studies, in this paper, we investigate blow-up results of problem (1). We will give the conditions for the blow-up results and establish the lower bounds for the blow-up rate. Our main results are as follows.

Theorem 1. Assume that $J_{1}\left(u_{0}\right)<0$. Then, the weak solution $u=u(x, t)$ of problem (1) blows up at finite time.

Theorem 2. Assume that $u_{0} \in H_{0}^{1}(\Omega)$ and $J_{1}\left(u_{0}\right)<0$, let $u=u(x, t)$ be the nonnegative solution of problem (1), then $u$ blows up in finite time

$$
T:=\frac{\left\|u_{0}\right\|_{2}^{2}}{-p(4 p+4) J_{1}\left(u_{0}\right)} .
$$

Theorem 3. Assume that $u_{0} \in H_{0}^{1}(\Omega), \quad J_{1}\left(u_{0}\right) \leq 0, \quad \int_{0}^{t_{0}}$ $\left\|u_{s}(\cdot, s)\right\|_{2}^{2} d s>0$ for any $t_{0}>0$, then, the weak solution $u=u(x, t)$ of problem (1) blows up at infinity. Moreover, if $\left\|u_{0}\right\|_{2} \leq\left(-J\left(u_{0}\right)\right)^{2 / p}$, the lower bound for blow-up rate can be estimated by

$$
\|u\|_{2}^{2} \geq\left\|u_{0}\right\|_{2}^{2}
$$

\section{Criterions of Blow-Up}

2.1. Preliminaries. In this section, we start with the definition of weak solution and blow-up at infinity of (1).

Definition 4 (weak solution). Let $T>0$. A function $u=$ $u(x, t) \in L^{\infty}\left(0, T ; X_{0}\right)$ with $u_{t} \in L^{p^{\prime}}\left(0, T ; W^{-1, p^{\prime}}(\Omega)\right) \cap L^{2}$ $\left(0, T ; L^{2}(\Omega)\right)$ is called a weak solution to problem (1) in $\Omega \times[0, T)$, if $u(x, 0)=u_{0}(x) \in X_{0}$ and $u(x, t)$ satisfies (1) in the sense of distribution, i.e.,

$$
\left\langle u_{t}, \omega\right\rangle+\left\langle|\nabla u|^{p-2} \nabla u, \nabla \omega\right\rangle+k\left\langle|u|^{p-2} u, \omega\right\rangle=\mu\left\langle u^{p} \int_{\Omega} u^{p+1}(y, t) d y, \omega\right\rangle,
$$

for all $\omega \in W_{0}^{1, p}(\Omega), t \in(0, T)$, where $X_{0}=W_{0}^{1, p}(\Omega) \backslash\{0\}$, $W^{-1, \tilde{p}}(\Omega)$ to denote the dual space of $W_{0}^{1, p}(\Omega)$, and $\tilde{p}$ is Holder conjugate exponent of $p>1$.

Definition 5 (blow-up at infinity). Let $u(x, t)$ be a weak solution of (1), we call $u(x, t)$ blow-up at $+\infty$ if the maximal existence time $T=+\infty$ and

$$
\lim _{t \longrightarrow+\infty}\|u(\cdot, t)\|_{2}=\infty
$$

To obtain the blow-up results, define the potential energy functional and the Nehari's functional as follows:

$$
\begin{aligned}
& J_{1}(u)=\frac{1}{p}\|\nabla u\|_{p}^{p}+\frac{k}{p}\|u\|_{p}^{p}-\frac{\mu}{2 p+2} \int_{\Omega} \int_{\Omega} u^{p+1}(x, t) u^{p+1}(y, t) d x d y \\
& I_{1}(u)=\|\nabla u\|_{p}^{p}+k\|u\|_{p}^{p}-\mu \int_{\Omega} \int_{\Omega} u^{p+1}(x, t) u^{p+1}(y, t) d x d y .
\end{aligned}
$$

To prove the main result, we need the following lemmas. 
Lemma 6. Assume that $u(x, t)$ is a weak solution of (1). Then, $J_{1}(u)$ is nonincreasing with respect to $t$ and satisfies the energy inequality

$$
\int_{0}^{t}\left\|u_{s}(\cdot, s)\right\|_{2}^{2} d s+J_{1}(u) \leq J_{1}\left(u_{0}\right), t \in\left[0, T_{0}\right)
$$

Proof. Similar to the proof in $[8,10,11]$, we can get the result.

Lemma 7. [8] $J_{1}(u)$ is a nonincreasing function, for $t \geq 0$,

$$
J_{l}^{\prime}(u)=-\left\|u_{t}\right\|^{2} \leq 0
$$

Lemma 8 [12]. Let $\Phi$ be a positive, twice differentiable function satisfying the following conditions:

$$
\begin{array}{r}
\Phi(\bar{t})>0, \\
\Phi^{\prime}(\bar{t})>0,
\end{array}
$$

for some $\bar{t} \in[0, T)$, and the inequality

$$
\Phi(t) \Phi^{\prime \prime}(t)-\alpha\left(\Phi^{\prime}(t)\right)^{2} \geq 0, \forall t \in[\bar{t}, T]
$$

where $\alpha>1$. Then, we have

$$
\Phi(t) \geq\left(\frac{1}{\Phi^{1-\alpha}(\bar{t})-\tilde{\Phi}(t-\bar{t})}\right)^{1 /(\alpha-1)}, t \in\left[t, T^{*}\right]
$$

in which $\tilde{\Phi}$ is a positive constant, and

$$
T^{*}=\bar{t}+\frac{\Phi(\bar{t})}{(\alpha-1) \Phi^{\prime}(\bar{t})}
$$

This implies

$$
\lim _{t \longrightarrow T^{*}} \Phi(t)=+\infty
$$

Lemma 9 [9]. Suppose that $\theta>0, \alpha>0, \beta>0$, and $h(t)$ is a nonnegative and absolutely continuous function satisfying $h$ ${ }^{\prime}(t)+\alpha h^{\theta}(t) \geq \beta$, then for $0<t<+\infty$, it holds

$$
h(t) \geq \min \left\{h(0),\left(\frac{\beta}{\alpha}\right)^{1 / \theta}\right\}
$$

2.2. Proof of the Main Results. We will consider the finite time blow-up results of problem (1) under the condition of nonpositive initial energy. The theorems are proved as follows.
Proof of Theorem 1. Assume that $u(x, t)$ is the weak solution of problem (1), for any $T>0$, we define the functional

$$
\Gamma(t)=\int_{0}^{t}\|u(\cdot, s)\|_{2}^{2} d s+(T-t)\left\|u_{0}\right\|_{2}^{2}, t \in[0, T] .
$$

It is obvious that $\Gamma(t)>0$ for all $t \in[0, T]$. Since $\Gamma$ is continuous, there exists $\rho>0$ (independent of $T$ ) such that $\Gamma(t)>\rho$ for all $t \in[0, T]$.

Then, we have

$$
\begin{aligned}
\Gamma^{\prime}(t) & =\|u(\cdot, t)\|_{2}^{2}-\left\|u_{0}\right\|_{2}^{2} \\
\Gamma^{\prime \prime}(t) & =2 \int_{\Omega} u_{t} u d x=-2 I_{1}(u) \geq-2 p J_{1}(u) .
\end{aligned}
$$

By using (12) in Lemma 7, we have

$$
-2 p J_{1}(u) \geq 2 p \int_{0}^{t}\left\|u_{s}(\cdot, s)\right\|_{2}^{2} d s-2 p J_{1}\left(u_{0}\right)
$$

From $J_{1}\left(u_{0}\right)<0$, (22) and (23), we get

$$
\Gamma^{\prime \prime}(t) \geq 2 p \int_{0}^{t}\left\|u_{s}(\cdot, s)\right\|_{2}^{2} d s-2 p J_{1}\left(u_{0}\right)>2 p \int_{0}^{t}\left\|u_{s}(\cdot, s)\right\|_{2}^{2} d s
$$

Now, multiplying (24) by $\Gamma(t)$, we have

$$
\begin{aligned}
\Gamma^{\prime \prime}(t) \Gamma(t)> & 2 p \int_{0}^{t}\left\|u_{s}(\cdot, s)\right\|_{2}^{2} d s \Gamma(t) \\
= & 2 p \int_{0}^{t}\left\|u_{s}(\cdot, s)\right\|_{2}^{2} d s \int_{0}^{t}\|u(\cdot, s)\|_{2}^{2} d s \\
& +2 p(T-t)\left\|u_{0}\right\|_{2}^{2} \int_{0}^{t}\left\|u_{s}(\cdot, s)\right\|_{2}^{2} d s
\end{aligned}
$$

Noticing that

$$
\begin{aligned}
\Gamma^{\prime}(t) & =\|u(\cdot, t)\|_{2}^{2}-\left\|u_{0}\right\|_{2}^{2} \\
& =\int_{0}^{t} \frac{d}{d s}\left(\|u(\cdot, s)\|_{2}^{2}\right) d s \\
& =2 \int_{0}^{t} \int_{\Omega} u_{s} u d x d s,
\end{aligned}
$$

then

$$
\left(\Gamma^{\prime}(t)\right)^{2}=4\left(\int_{0}^{t} \int_{\Omega} u_{s} u d x d s\right)^{2} .
$$

With the help of Cauchy-Schwarz inequality, we have

$$
\left(\Gamma^{\prime}(t)\right)^{2} \leq 4 \int_{0}^{t}\left\|u_{s}(\cdot, s)\right\|_{2}^{2} d s \int_{0}^{t}\|u(\cdot, s)\|_{2}^{2} d s
$$


Using (25) and (28), we further get

$\Gamma^{\prime \prime}(t) \Gamma(t)-\frac{p}{2}\left(\Gamma^{\prime}(t)\right)^{2}>2 p(T-t)\left\|u_{0}\right\|_{2}^{2} \int_{0}^{t}\left\|u_{s}(\cdot, s)\right\|_{2}^{2} d s>0$,

for all $t \in[0, T]$.

By Lemma 8 , there exists $T^{*}>0$ such that

$$
\lim _{t \longrightarrow T^{*}} \Gamma(t)=+\infty
$$

which implies

$$
\lim _{t \rightarrow T^{*}} \int_{0}^{t}\|u(\cdot, s)\|_{2}^{2} d s=+\infty
$$

As a consequence, we get

$$
\lim _{t \longrightarrow T^{*}}\|u(\cdot, t)\|_{2}^{2} d s=+\infty
$$

this means $u(x, t)$ blows up at finite time $T^{*}$.

Proof of Theorem 2. Set $G(t)=\|u(\cdot, s)\|_{2}^{2}$, then

$$
\begin{aligned}
G^{\prime}(t) & =2 \int_{\Omega} u_{t} u d x \\
& =2\left(-\|\nabla u\|_{p}^{p}+\mu \int_{\Omega} \int_{\Omega} u^{p+1}(x, t) u^{p+1}(y, t) d x d y-k\|u\|_{p}^{p}\right) \\
& =-2 I_{1}(u) \\
& \geq F(t)
\end{aligned}
$$

in which

$$
\begin{aligned}
F(t):= & -(4 p+4) J_{1}(u) \\
= & -\frac{4 p+4}{p}\|\nabla u\|_{p}^{p}-\frac{k(4 p+4)}{p}\|u\|_{p}^{p} \\
& +2 \mu \int_{\Omega} \int_{\Omega} u^{p+1}(x, t) u^{p+1}(y, t) d x d y .
\end{aligned}
$$

By Lemma 6, we can get

$$
F^{\prime}(t)=-(4 p+4) \frac{d}{d t} J_{1}(u)=(4 p+4) \int_{\Omega}\left|u_{t}\right|^{2} d x>0 .
$$

From (35) and the condition $J_{1}\left(u_{0}\right)<0$, we can get $F(t)>0$. Then, by Cauchy-Schwarz inequality and (21), the following inequality can be obtained:

$$
\begin{aligned}
G(t) F^{\prime}(t) & =(4 p+4) \int_{\Omega}|u|^{2} d x \int_{\Omega}\left|u_{t}\right|^{2} d x \\
& \geq(4 p+4)\left(\int_{\Omega} u u_{t} d x\right)^{2} \\
& =(p+1)\left[G^{\prime}(t)\right]^{2} \\
& \geq(p+1) G^{\prime}(t) F(t),
\end{aligned}
$$

that is,

$$
\frac{F^{\prime}(t)}{F(t)} \geq(p+1) \frac{G^{\prime}(t)}{G(t)}
$$

Integrating (37) on $[0, t]$, we can get

$\ln [F(t)]-\ln [F(0)] \geq(p+1) \ln [G(t)]-(p+1) \ln [G(0)]$,

that is,

$$
\frac{F(t)}{[G(t)]^{p+1}} \geq \frac{F(0)}{[G(0)]^{p+1}} .
$$

By (33), we have

$$
\frac{G^{\prime}(t)}{[G(t)]^{p+1}} \geq \frac{F(0)}{[G(0)]^{p+1}}
$$

By integrating (40) on $[0, t]$, we can get

$$
\frac{1}{[G(t)]^{p}} \leq \frac{1}{[G(0)]^{p}}-\frac{p F(0)}{[G(0)]^{p+1}} t=\frac{G(0)-p F(0) t}{[G(0)]^{p+1}} .
$$

Take the reciprocal of (41) to get

$$
[G(t)]^{p} \geq \frac{[G(0)]^{p+1}}{G(0)-p F(0) t} .
$$

Let

$$
T=\frac{G(0)}{p F(0)}=\frac{\left\|u_{0}\right\|_{2}^{2}}{-p(4 p+4) J_{1}\left(u_{0}\right)},
$$

when $t \longrightarrow T^{-}$, we can get $[G(t)]^{p} \longrightarrow+\infty$; this means that $u$ blows up in a finite time.

Proof of Theorem 3. Assume that $u(x, t)$ is the weak solution of problem (1). Set $G(t)=\|u(\cdot, s)\|_{2}^{2}$, then

$$
G^{\prime}(t)=2 \int_{\Omega} u_{t} u d x=-2 I_{1}(u) \geq-2 p J_{1}(u)
$$

By (12) in Lemma 6 and the condition $J_{1}\left(u_{0}\right) \leq 0$, we can get 


$$
-2 p J_{1}(u) \geq 2 p \int_{0}^{t}\left\|u_{s}(\cdot, s)\right\|_{2}^{2} d s .
$$

Then, by (44) and (45), we have

$$
G^{\prime}(t) \geq 2 p \int_{0}^{t}\left\|u_{s}(\cdot, s)\right\|_{2}^{2} d s>0
$$

Fix $t_{0}>0$ and let $\kappa=\int_{0}^{t}\|u\|_{2}^{2} d s$. By the condition $\int_{0}^{t_{0}}\left\|u_{s}(\cdot, s)\right\|_{2}^{2} d s>0$, we can get $\kappa$, which is a positive constant. Integrating $(46)$ over $\left(t_{0}, t\right)$, we can get

$$
\begin{aligned}
G(t) & \geq G\left(t_{0}\right)+2 p \int_{t_{0}}^{t} \int_{0}^{t}\left\|u_{s}(\cdot, s)\right\|_{2}^{2} d s d \tau \\
& \geq G\left(t_{0}\right)+2 p \int_{t_{0}}^{t} \kappa d \tau \\
& \geq 2 p \kappa\left(t-t_{0}\right) .
\end{aligned}
$$

Hence, we have

$$
\lim _{t \longrightarrow+\infty} G(t)=\infty
$$

This means that the weak solution $u=u(x, t)$ of problem (1) blows up at infinity.

From (12) and (44), we have

$$
\begin{aligned}
G^{\prime}(t) & \geq-2 p J_{1}(u) \geq-2 p J_{1}\left(u_{0}\right), \\
G^{\prime}(t)+G^{p / 2}(t) & \geq-2 p J_{1}\left(u_{0}\right) \geq-J_{1}\left(u_{0}\right),
\end{aligned}
$$

for $p>2$.

By Lemma 9, consider $J_{1}\left(u_{0}\right)<0$ and $\left\|u_{0}\right\|_{2}^{2} \leq$ $\left(-J_{1}\left(u_{0}\right)\right)^{2 / p}$, we have

$$
G(t) \geq \min \left\{\left\|u_{0}\right\|_{2}^{2},\left(-J_{1}\left(u_{0}\right)\right)^{2 / p}\right\} \geq\left\|u_{0}\right\|_{2}^{2},
$$

this ends the proof.

\section{Conclusions}

In this work, we consider the initial boundary value problem for a class of quasilinear parabolic equation with power nonlinearity and nonlocal source under homogeneous Dirichlet boundary condition in a smooth bounded domain. Some new results of blow-up and blow-up time under the condition of nonpositive initial energy are obtained. The blowup results of problem (1) with arbitrary initial energy will be the direction of further research.

\section{Data Availability}

No data were used to support this study.

\section{Conflicts of Interest}

The authors declare that there is no conflict of interests regarding the publication of this paper.

\section{Authors' Contributions}

Xiaorong Zhang and Zhoujin Cui contributed equally to this work. All authors carried out the proof and conception of the study. All authors read and approved the final manuscript.

\section{Acknowledgments}

This project is supported by the Major Projects of Natural Sciences of University in Jiangsu Province of China (No. 18KJA110003) and the Qianfan Plan of Jiangsu Maritime Institute (No. 014060).

\section{References}

[1] P. Souplet, "Uniform blow-up profiles and boundary behavior for diffusion equations with nonlocal nonlinear source," Journal of Differential Equations, vol. 153, no. 2, pp. 374-406, 1999.

[2] K. Bimpong-Bota, P. Ortoleva, and J. Ross, "Far-from-equilibrium phenomena at local sites of reaction," The Journal of Chemical Physics, vol. 60, no. 8, pp. 3124-3133, 1974.

[3] V. A. Galaktionov, "On asymptotic self-similar Behaviour for a quasilinear heat equation: single point blow-up," SIAM Journal on Mathematical Analysis, vol. 26, no. 3, pp. 675-693, 1995.

[4] A. A. Samarskii, S. P. Kurdyumov, V. A. Galaktionov, and A. P. Mikhailov, Blow-Up in Problems for Quasilinear Parabolic Equations (in Russian), Nauka, Moscow, (1987), Walter de Gruyter, Berlin, 1995.

[5] J. Furter and M. Grinfeld, "Local vs. non-local interactions in population dynamics," Journal of Mathematical Biology, vol. 27, no. 1, pp. 65-80, 1989.

[6] R. S. Cantrell and C. Cosner, "Diffusive logistic equations with indefinite weights: population models in disrupted environments II," SIAM Journal on Mathematical Analysis, vol. 22, no. 4, pp. 1043-1064, 1989.

[7] F. C. Li and C. H. Xie, "Global and blow-up solutions to a p-Laplacian equation with nonlocal source," Computers of Mathematcs with Applications, vol. 46, no. 10-11, pp. 15251533, 2003.

[8] S. Boulaaras, A. Choucha, A. Zara, M. Abdalla, and B. B. Cheri, "Global existence and decay estimates of energy of solutions for a new class of $p$-Laplacian heat equations with logarithmic nonlinearity," Journal of Function Spaces, vol. 2021, Article ID 5558818, 11 pages, 2021.

[9] S. Toualbia, A. Zaraï, and S. Boulaaras, "Decay estimate and non-extinction of solutions of p-Laplacian nonlocal heat equations," AIMS Mathematics, vol. 5, no. 3, pp. 1663-1679, 2020.

[10] H. Ding and J. Zhou, "Global existence and blow-up for a mixed pseudo-parabolic $p$-Laplacian type equation with logarithmic nonlinearity," Journal of Mathematical Analysis and Applications, vol. 478, no. 2, pp. 393-420, 2019.

[11] G. Liu and H. Zhang, "Blow-up phenomena for a class of parabolic or pseudo-parabolic equation with nonlocal source," Mediterranean Journal of Mathematics, vol. 18, no. 3, p. 85, 2021.

[12] C. N. le and X. T. Le, "Global solution and blow-up for a class of $p$-Laplacian evolution equations with logarithmic nonlinearity," Acta Applicandae Mathematicae, vol. 151, no. 1, pp. 149-169, 2017. 
[13] Y. He, H. Gao, and H. Wang, "Blow-up and decay for a class of pseudo-parabolic p-Laplacian equation with logarithmic nonlinearity," Computers \& Mathematcs with Applications, vol. 75, no. 2, pp. 459-469, 2018.

[14] Z. Cui and Z. Yang, "A Neumann problem for quasilinear elliptic equations with logarithmic nonlinearity in a ball," Complex Variables and Elliptic Equations, vol. 55, no. 12, pp. 1143-1153, 2010.

[15] Z. Cui, Z. Mao, W. Zong, X. Zhang, and Z. Yang, "Existence results for a class of the quasilinear elliptic equations with the logarithmic nonlinearity," Journal of Function Spaces, vol. 2020, Article ID 6545918, 9 pages, 2020.

[16] G. Astrita and G. Marrucci, Principles of Non-Newtonian Fluid Mechanics, McGraw-Hill, New York, 1974.

[17] L. K. Martinson and K. B. Pavlov, "Unsteady shear flows of a conducting fluid with a rheological power law," Akademiya Nauk Latviiskoy SSR, vol. 2, pp. 50-58, 1971.

[18] A. S. Kalashnikov, "On a nonlinear equation appearing in the theory of non-stationary filtration," Moskovskiy Universitet, vol. 5, pp. 60-68, 1978.

[19] Z. Yang and Q. Lu, "Nonexistence of positive solutions to a quasilinear elliptic system and blow- up estimates for a nonNewtonian filtration system," Applied Mathematics Letters, vol. 16, no. 4, pp. 581-587, 2003.

[20] J. R. Esteban and J. L. Vazquez, "On the equation of turbulent filtration in one-dimensional porous media," Nonlinear Analysis, vol. 10, pp. 1303-1325, 1982. 